\title{
Modeling on Single Abrasive Lapping Force in Through-feed Super-finishing of Tapered Rollers
}

\author{
Xue Jinxue ${ }^{1, a}$, Zhang Ying ${ }^{1, b}$,Wang Lina ${ }^{1, c}$ and Wang Fang ${ }^{1, d}$
}

${ }^{1}$ School of Mechatronics Engineering, Henan University of Science \& Technology, Luoyang, China

axjx19652000@163.com, bzhangypds@163.com, cwanglinazs@163.com, wfcitic@126.com

Keywords: tapered roller, through-feed super-finishing, abrasive grain, lapping force model Abstract. Through-feed super-finishing is the main processing technology to roller convexity. The research on lapping force of tapered rollers in through-feed super-finishing is the key to further study the material removal mechanism of the roller. Taking a single grain as the object, the movement characteristics of abrasive grains in the through-feed super-finishing were obtained. The abrasive lapping force model was established on the basis of the cutting mechanism and kinematic analysis of through-feed super-finishing. The influence tendencies of different processing parameters on the lapping force were analyzed qualitatively, which provide theoretical foundation to the further study of tapered rollers machining.

\section{Introduction}

Rolling bearing is a kind of important and basic part in machinery, and tapered roller is a kind of important rolling element [1]. Tapered roller's convexity modification can avoid or mitigate the edge stress concentration, and make the contact stress distribution tend to be uniform [2], Through-feed super-finishing is the main processing technology to machine the roller convexity and determines the final surface quality of the tapered rollers.

Because of the particularity of the through-feed super-finishing lapping motion of tapered roller, the lapping force needs to be systematically analyzed [3]. The single abrasive grains in through-feed super-finishing oilstone strips were analyzed in this paper. The lapping force had been analyzed qualitatively. Therefore, it is of great practical significance to analyze the single abrasive lapping force in through-feed super-finishing of tapered rollers.

\section{The Processing Method of Though-feed Super-finishing}

Fig. 1 shows the processing method of through-feed super-finishing of taper rollers by oilstone. Under the driving action of the friction support and the spiral edge of the front and back guide roller, the tapered roller runs forward and rotates at a certain speed. The oilstone presses the roller at a certain pressure and moves at high frequency oscillation. Based on discrete idea, the tapered rollers to be researched will be divided into several small zones to get the mathematical model.
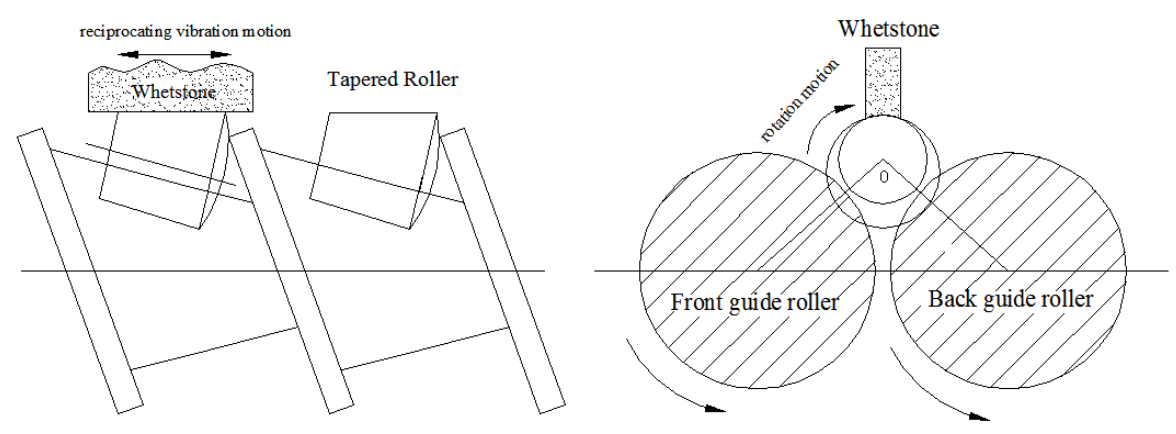

Fig.1 Schematic diagram of tapered roller through-feed super-finishing processing 


\section{Geometric Analysis of Contact Arc length of Single Abrasive Grain}

The length of contact arc between oilstone and roller in lapping is one of the most important parameters in the process. It is almost related to all the lapping parameters, especially the lapping temperature, lapping force and surface contour of the roller after lapping. According to the principle of relative motion, motion diagram of the single abrasive grain in through-feed super-finishing was shown in fig.2.
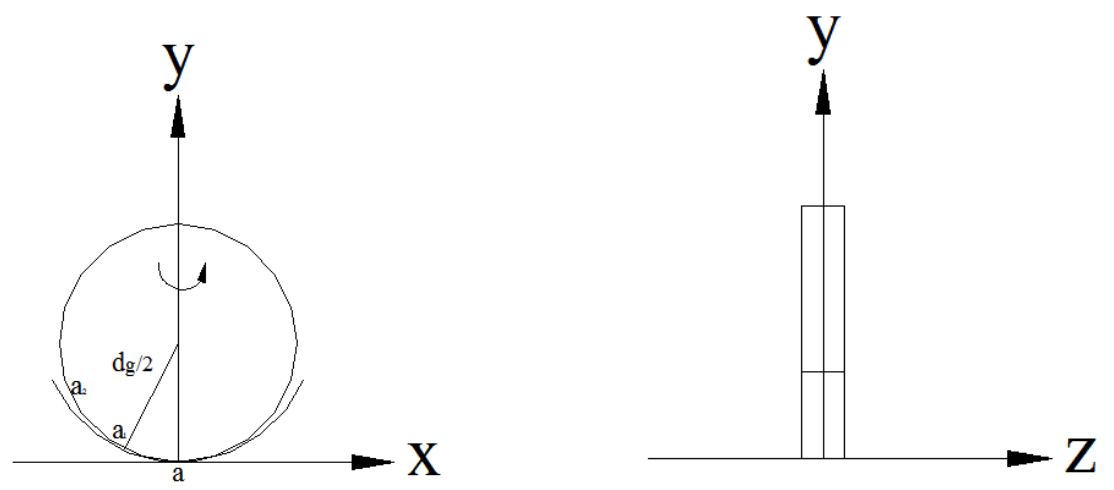

Fig.2 Contact geometry sketch between roller and oilstone

If the roller was stationary, the micro cutting motion of the abrasives is converted to motion in a circle with a certain radius. The locus equation of abrasive grains can be given as

$$
\left\{\begin{array}{l}
x=\frac{d_{g}}{2} \sin \left(\omega_{g} t\right) \\
y=\frac{d_{g}}{2}-\frac{d_{g}}{2} \cos \left(\omega_{g} t\right) . \\
z=v_{a} t \pm A t \sin (2 \pi f t)
\end{array}\right.
$$

where, $A$ represents amplitude of oilstone, $f$ represents oscillation frequency of oilstone, $v_{a}$ represents axial feed rate of rollers, $\omega_{g}$ represents spin velocity of tapered roller, $d_{g}$ represents tapered roller diameter, and $t$ represents single grain micro cutting time.

The contact arc length of a single abrasive can be obtained according to Eq.1. $\Delta t$ represents total time of abrasive micro cutting. It can be obtained by analyzing the relationship between the cutting depth and the geometry of the motion. $\Delta t$ can be given as

$$
\Delta t=\sqrt{\frac{a_{g} d_{g}}{v_{g}}} .
$$

where, $a_{g}$ represents lapping depth, $v_{g}$ represents rotation liner speed of the tapered roller.

Coefficient $k(k>1)$ is added to Eq.3, which can be obtained by experiments. The influence of $v_{a}$ on $l_{k}$ can be ignored. According to above conclusions, the equation of $l_{k}$ is as follows,

$$
l_{k}=k \sqrt{\frac{a_{g} d_{g}}{v_{g}}} \sqrt{\left(\omega_{g} \frac{d_{g}}{2}\right)^{2}+(4 A f)^{2}} .
$$

According to references [2], the key parameters in Eq. 3 can be determined as followed: $k=1.35$, $A=2 \mathrm{~mm}, f=46 \mathrm{~Hz}, \omega_{g}=100 \mathrm{rad} / \mathrm{s}$. Fig. 4 and fig. 5 show the simulation results utilizing MATLAB. 


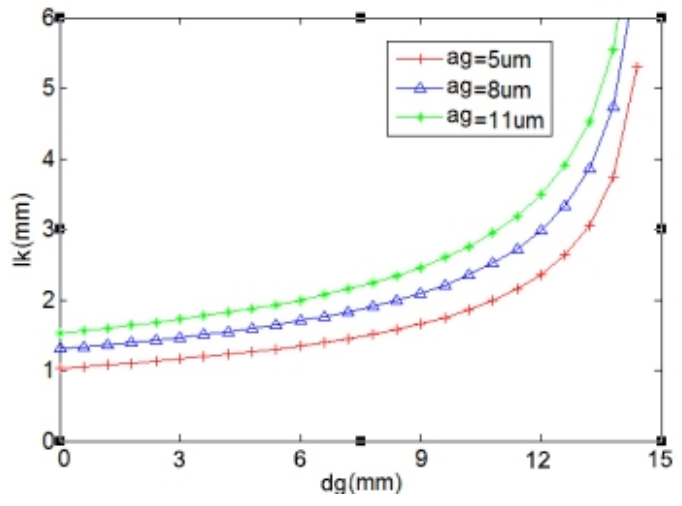

Fig. 3 The influence of $a_{g}$ to $l_{k}$

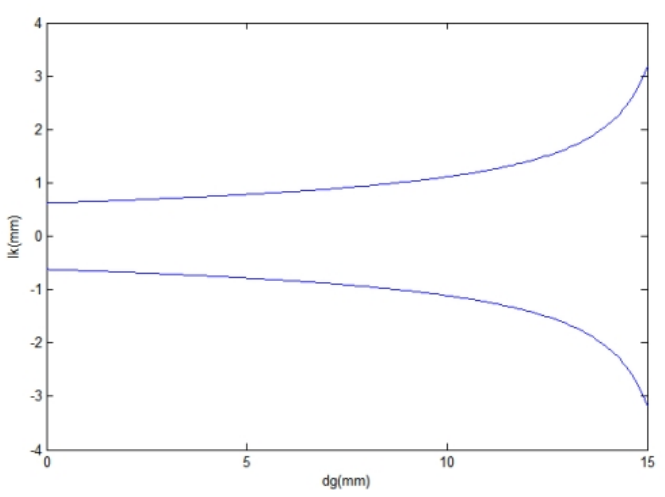

Fig.4 Contact curve range

It can be seen from fig. 3 that the contact arc length of the single abrasive increases with the increase of the radius of the roller slice. The increasing rate was relatively small in small end of the roller, but when closer to the large end the increasing rate is higher relatively. From Fig. 4 it can be concluded that the contact arc length is distributed on the tapered roller. The contact curve was symmetrically distributed on both sides of the generating line .There are contact line with the pattern of " $\pi$ " in the lapping area. The above analysis show that the grind amount at both ends of the roller is greater than that at the middle part, which is beneficial to the formation of convexity.

\section{Establishment of Dynamic Model of Effective Grains ' Number}

The distribution of grains on the oilstone is random. In this paper, it was assumed that the distribution of abrasive grains and the height of the cutting edge conform to the normal distribution. The size of abrasive grain is the basis of calculating the total dynamic effective lapping number. Grain size refers to the geometric size of grains. The size of the abrasive grains is divided into 41 categories according to its geometric size. The grain size can be obtained by table 1[4].

Table 1 Size of abrasive grain

\begin{tabular}{c|cccc}
\hline Grain size & W50 & W40 & W28 & W20 \\
\hline Nominal dimension $[\mu \mathrm{m}]$ & $50 \sim 40$ & $40 \sim 28$ & $28 \sim 20$ & $20 \sim 14$ \\
\hline Grain size & W14 & W10 & W7 & W5 \\
\hline Nominal dimension $[\mu \mathrm{m}]$ & $14 \sim 10$ & $10 \sim 7$ & $7 \sim 5$ & $5 \sim 3.5$ \\
\hline
\end{tabular}

Table 2 Structure number of oilstone and worn grains ratio

\begin{tabular}{c|ccccccc}
\hline $\begin{array}{c}\text { structure } \\
\text { number }\end{array}$ & 1 & 2 & 3 & 4 & 5 & 6 & 7 \\
\hline $\begin{array}{c}\text { worn grains } \\
\text { ratio }\end{array}$ & $62 \%$ & $60 \%$ & $58 \%$ & $56 \%$ & $54 \%$ & $52 \%$ & $50 \%$ \\
\hline
\end{tabular}

Because of the complex shape of abrasive grains, the abrasive grains can be assumed to be spheres for easy analysis. Abrasive grains are tightly bonded together by resin bond. $\Psi$ represents worn grain ratio. Worn grain ratio refers to the percentage of grain in the oilstone. The worn grain ratio can be obtained by table 2[4].Because the lapping process does not involve all abrasive grains, a lapping coefficient $Q$ should be introduced. The calculating equation of dynamic effective grains ' number is as follows,

$$
N_{z}=\operatorname{Qnbl}\left(\frac{8 \psi}{\pi d^{3}}\right)^{\frac{2}{3}} .
$$

where, $l$ represents the length of contact arc and $b$ represents the contact width. The model of dynamic effective grains' numbers is analyzed in this section, which is derived from the total number and the 
number of abrasive grain involved in cutting. The obtained model is more consistent with the actual lapping process.

\section{Calculation and Analysis of Single Abrasive Lapping Force}

Assuming that the equivalent mass of the oilstone is $M$, the single abrasive grain force acting on the oilstone in the through-feed super-finishing process of the roller is as follows

$$
F_{m d}=\frac{F_{m}}{N_{d}}=\frac{M A \omega \cos \omega t}{Q B l\left(\frac{8 \psi}{\pi d_{\text {mean }}^{3}}\right)^{\frac{2}{3}} \Delta t}
$$

Assuming that the load of a single abrasive grain on the oilstone is $H$, the lapping force of the single abrasive grain is

$$
F_{y d}=\frac{H}{N_{d}}=\frac{H}{Q B l\left(\frac{8 \psi}{\pi d_{\text {mean }}^{3}}\right)^{\frac{2}{3}} \Delta t .}
$$

In summary, the lapping force on a single abrasive grain is derived. it can be given as

$$
F_{z d}=F_{m d}+F_{y d}=\frac{M A \omega \cos \omega t+H}{Q B l\left(\frac{8 \psi}{\pi d_{\text {mean }}^{3}}\right)^{\frac{2}{3}} \Delta t} .
$$

Through the analysis of the lapping force model, the lapping force is increased with the smaller worn grain ratio and larger average radius of the grain. When the worn grain ratio and average radius are constant, the lapping force of the single abrasive grain is increased with the smaller length and width of the oilstone.

\section{Conclusions}

The lapping mechanism and kinematics of through-feed super-finishing of tapered rollers are analyzed in this paper, and the conclusions are as follows:

1 The contact arc length of single abrasive grain increases with the roller diameter and cutting depth of abrasive grain of oilstone.

2 Based on the model of dynamic effective grains ' number, the single abrasive lapping force in through-feed super-finishing of tapered rollers is established. When the average diameter of the abrasive grain is larger and the worn grain ratio is smaller, better processing effect can be obtained.

\section{Acknowledgments}

This research derives from the National Natural Science Foundation in China (No. 51475145).

\section{References}

[1] GAO Zuo-bin, MA Wei, DENG Xiao-zhong: Research Progress on Convexity Machining Technology for Roller Bearings. Bearing. 10 (2011), p. 61-63

[2] Xue Jin-xue, JIA Song-yang, YANG Bai-song: The polish properties analysis of though-feed superfinishing tapered roller. Manufacturing Automation. 38-1 (2016), p. 33-37

[3] Zheng Hongwei, He Hongxia, He Meng: Determination of Superfinishing Process Parameters for Cylindrical Roller D42218N3W.Bearing . 10 (2014), p. 16-18

[4] Li Bomin, Zhao Bo, Li Qing:Abrasives material, abrasive tools and lapping technology, edtied by Chemical Industry Press, Beijing (2004). 